\title{
Fundamental Experiments to Develop Eco-friendly Techniques for Conserving Frog Habitat in Paddy Areas: Escape Countermeasures for Frogs Falling into Agricultural Concrete Canals
}

\author{
Keiji WATABE*, Atsushi MORI, Noriyuki KOIZUMI and \\ Takeshi TAKEMURA \\ Department of Rural Environment, National Institute for Rural Engineering, \\ National Agriculture and Food Research Organization (Tsukuba, Ibaraki 305-8609, Japan)
}

\begin{abstract}
Frogs often drown in agricultural canals with deep concrete walls that are installed commonly in paddy areas after land consolidation projects in Japan because they cannot escape after falling into the canal. We propose a partial concrete canal with gently sloped walls as a countermeasure for frogs to escape the canal and investigated the preferable angle of the sloped walls, water depth and flow velocity for Rana porosa porosa. Our experiments showed that only 13 individuals (2\%) escaped by leaping out of the canal, indicating that climbing up is the main escape behavior of $R$. p. porosa. Walls with slopes of $30-45$ degrees allowed $50-60 \%$ of frogs to escape from experimental canals, the frogs especially easily climbed up walls with a 30 degree slope. Adjusting water depth to $5 \mathrm{~cm}$ or more would assist the frogs in reaching the escape countermeasures because at such depths frogs are not able to stand on the canal bottom and to move freely about. Flow velocity should be slower around the countermeasures because $R$. p. porosa is not good at long-distance swimming and cannot remain under running water for a long time. The broadened inlets of this new canal design provide water of slower velocity near the shores of the sloped walls, which encourages frogs to escape.
\end{abstract}

Discipline: Agricultural environment

Additional key words: climbing capability, ecosystem conservation, Japan, Rana porosa porosa, Tokyo Daruma Pond Frog

\section{Introduction}

Frogs are important bioindicators, a sort of barometer of the environmental health because they are in high environmental demand and occupy an intermediary position in food webs ${ }^{13}$. Both aquatic habitats and terrestrial habitats are necessary for many species of frogs: tadpoles live in the water and metamorphosed frogs live on the shore. On the other hand, they are consumers of small animals such as insects and spiders ${ }^{6}$, and are also important diets of large predatory animals such as freshwater fish, snakes, herons, and Japanese minks in both aquatic and terrestrial food webs ${ }^{4,9,10,13}$. They are particularly sensitive to environmental stresses such as habitat loss, pollutants, ultraviolet light, alien species, infections, and climate change ${ }^{13}$. Approximately 32\% of some 6,000 amphibian species including frogs and salamanders are pres- ently threatened with extinction ${ }^{19}$ because of increasing environmental stresses related to human activities such as deforestation, chemical pollution, acid precipitation, and other unknown causes. Conservation countermeasures are being considered and implemented worldwide to protect amphibians from extinction, and numerous volunteers in Western Europe, North and Central America, and Australia are taking personal measures by managing and creating frog habitats. Countries such as the United States, Denmark, France, and Germany have enacted legislation to protect amphibians, and the Ministry of Agriculture, Forestry and Fisheries in Japan provides extra subsidies to local governments and farmers' organizations for the construction of amphibian-friendly agricultural canals.

Twelve frog species inhabit paddy fields and agricultural canals in Japan (Table 1) $)^{5,11}$. Hyla japonica, Rana japonica, Rana limnocharis, and Rhacophorus schlegelii 
are common species across the islands of Japan, and Rana porosa porosa, Rana porosa brevipoda and Rana nigromaculata are regionally common species. Some species of frogs such as H. japonica tree frogs (Fig. 1a) have adhesive discs, whereas $R$. p. porosa lack discs (Fig. 1b). Rana p. porosa is a common species on the Kanto Plain, where a modern farming system of paddy and vegetable fields has been developed around the large metropolitan area of Tokyo. The habitat and nursery-site functions of the Kanto Plain have been degraded by rapid development and agricultural modernization such as usage of pesticides and land improvement projects, including land consolidation and the replacement of shallow earthen canals with concrete irrigation and drainage canals. The new irrigation canals are separated from drainage canals; water is typically pumped into paddy fields via under-

Table 1. Frogs that live in Honshu, Shikoku and Kyushu Islands, Japan

\begin{tabular}{|c|c|c|}
\hline Common name & Nomenclature & Main spawning sites \\
\hline Eastern-Japanese Common Toad & Bufo japonicus formosus & Paddy fields, temporary puddles \\
\hline Western-Japanese Common Toad & B. j. japonicus & Paddy fields, temporary puddles \\
\hline Japanese Stream Toad & B. torrenticola & Pools of mountain streams \\
\hline Japanese Tree Frog & Hyla japonica & Paddy fields, temporary puddles \\
\hline Japanese Brown Frog & Rana japonica & Paddy fields \\
\hline Montane Brown Frog & R. ornativentris & Paddy fields, temporary puddles \\
\hline Black-spotted Pond Frog & R. nigromaculata & Paddy fields \\
\hline Tokyo Daruma Pond Frog & R. porosa porosa & Paddy fields \\
\hline Daruma Pond Frog & R. p. brevipoda & Paddy fields \\
\hline Wrinkled Frog & R. rugosa & Paddy fields, small streams, mountain streams \\
\hline Indian Rice Frog & R. limnocharis & Paddy fields \\
\hline Tago's Brown Frog & R. tagoi tagoi & Mountain streams \\
\hline Stream Brown Frog & R. sakuraii & Pools of mountain streams \\
\hline Forest Green Tree Frog & Rhacophorus arboreus & Ponds, paddy fields \\
\hline Schlegel's Green Tree Frog & Rh. schlegelii & Paddy fields \\
\hline Kajika Frog & Buergeria buergeri & Shallows of mountain streams \\
\hline
\end{tabular}

Made by Hasegawa (2003) ${ }^{5}$

a)

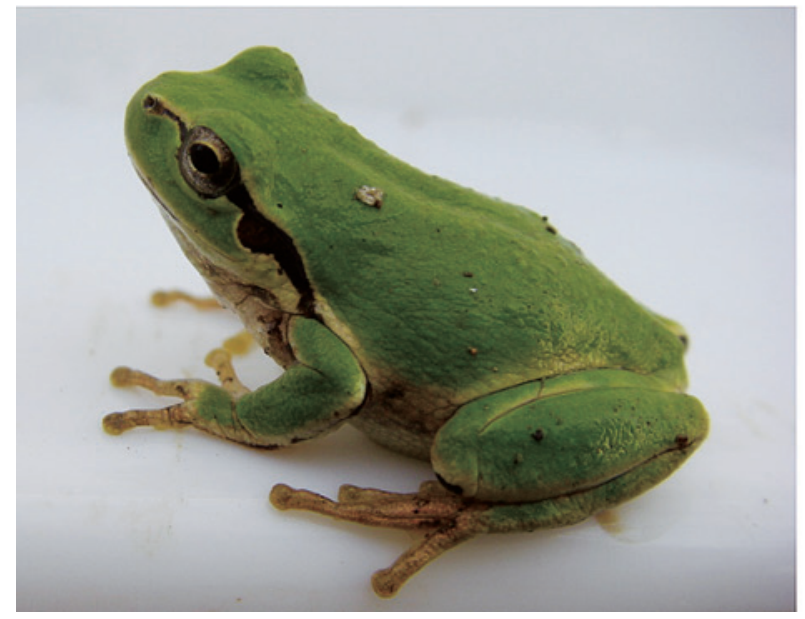

b)

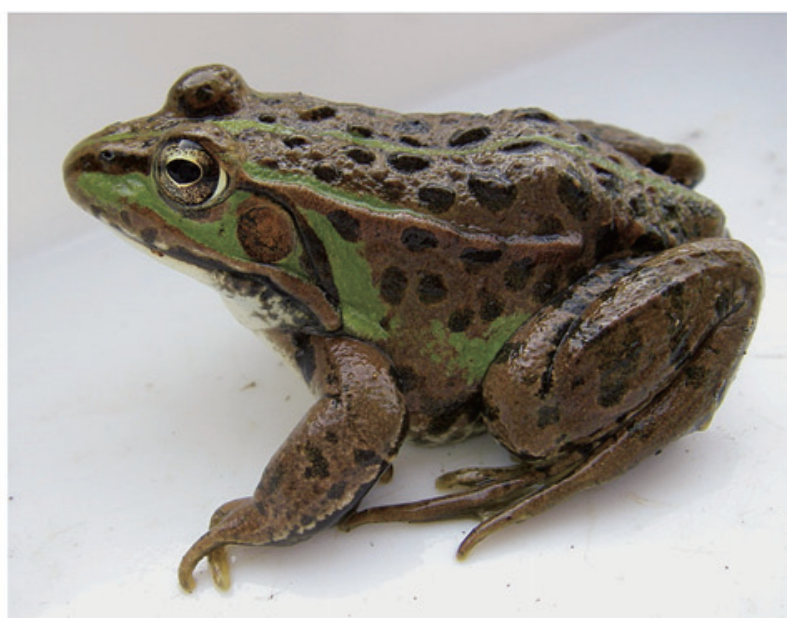

Fig. 1. Frogs that live in paddy areas

a): Hyla japonica the Japanese Tree Frog that has adhesive discs.

b): Rana porosa porosa the Tokyo Daruma Pond Frog that lacks adhesive discs. 
ground pipes and drained from fields into deep, Ushaped, concrete canals in which the water level may be $70 \mathrm{~cm}$ or more below that of the rice fields ${ }^{3}$. The typical U-shaped canal consists of a concrete channel (width 50$80 \mathrm{~cm}$ ) with vertical sides (height 60-90 cm) ${ }^{3}$, and water depth and velocity in the canals change seasonally from 0 to $20 \mathrm{~cm}$ and from around 0 to $10 \mathrm{~cm} / \mathrm{s}$, respectively. The changes in habitats caused by the projects, especially the replacement of earthen canals with concrete canals, may be one of the factors causing frog population declines in Japan. Fujioka \& Lane ${ }^{3}$ reported that only few frogs lacking adhesive discs live in the paddy fields after land improvement projects. Matsuzawa et al. ${ }^{12}$ reported that $R h$. schlegelii individuals, which have adhesive discs, often drown in concrete agricultural canals with a width of 30 $\mathrm{cm}$. These observations indicate that frogs falling into the concrete canals cannot escape and are washed downstream and drown in the canals.

Countermeasures to compensate for changing canals or as part of environmental improvement activities by farmers are necessary to enable frogs to move freely across agricultural canals and escape easily once they enter the canals. Mizutani et al. ${ }^{14}$ reported the effectiveness of placing a lid on canals to prevent frogs from falling into canals. Existing canal designs that enable frogs to escape are broadly classified into types A and B. Type A canals contain a ramp sloped toward the opposite direction of flow in the canals (Fig. 2a). This type cannot help frogs to escape if they are washed out through the main flow. The nearly vertical walls of type $B$ canals have a rough surface to assist frogs in escaping (Fig. 2b). Such nearly vertical walls mean type B canals take less space in the fields, but frogs cannot easily reach the rough surfaces. Neither type A nor type B canals have components that reduce water flow and allow pools or inlets to form. Therefore, we propose a design for type C canals, which have some wider areas with deeply sloped walls (Fig. 3). The design of type $\mathrm{C}$ canals allows reduced water flow in these wider areas, which form artificial inlets with shallow water depth, and slow water flow along each sloped wall should make it easier for frogs to reach the sloped wall and escape.

A successful design for type $\mathrm{C}$ canals must take into consideration the behavior and physical characteristics of frogs. However, this information has hardly been studied with only a few reports on climbing ability and jumping ability ${ }^{1,2,16}$. Of this information, the hydraulic condition of the canal, as well as the angle of sloped walls as a coun-



Fig. 3. Proposed type C escape countermeasure with placement of partially concrete sloped walls toward the vertical direction of flow a)

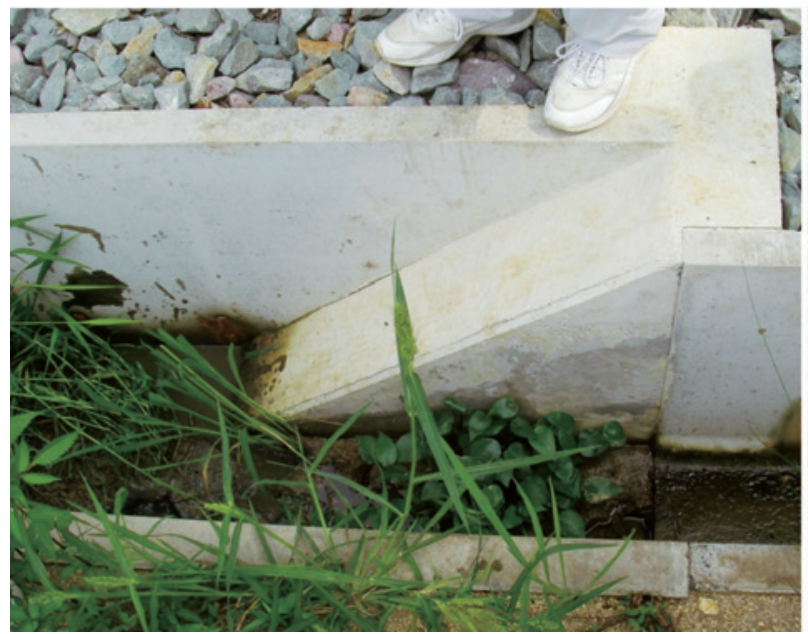

b)

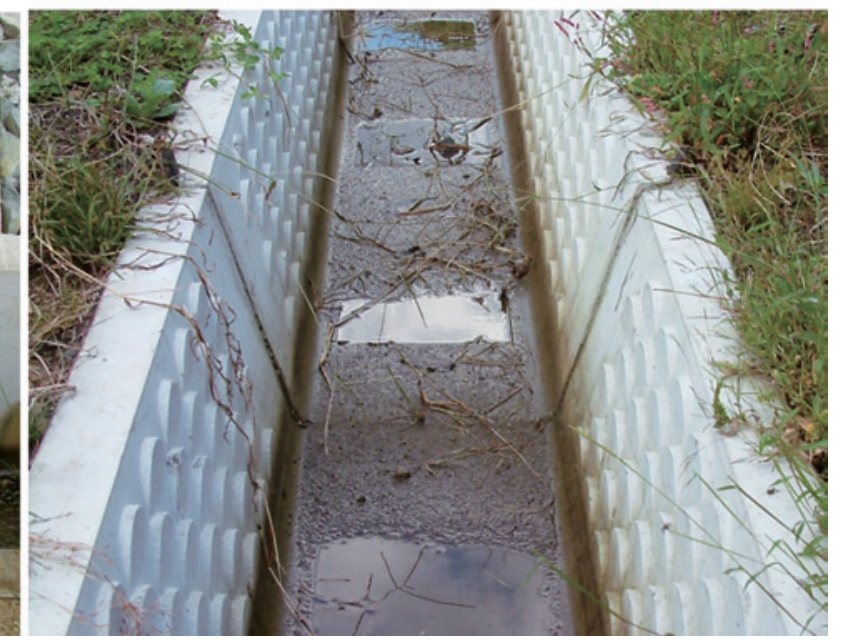

Fig. 2. Example of the escape countermeasures for frogs

a): Placement of partially sloped walls in the canal, type A.

b): Placement of rough surfaces on sloped walls of the canal, type B. 
termeasure that enables frogs to escape easily, was focused on because water depth and flow velocity of canals may affect frog behavior until getting on the countermeasure.

In this study, R. p. porosa the Tokyo Daruma Pond Frog (Fig. 1b) was targeted because this frog is now designated as a "Near Threatened" species by the Ministry of the Environment. Even though they were common species decades ago, they are now threatened because of habitat loss by rapid development, changing earth canals into canals with deep vertical concrete walls, usage of pesticides etc. They are especially sensitive to the canal improvements of land improvement projects because they have no adhesive discs and cannot escape from canals with deep concrete walls ${ }^{3,17}$. The objective of this study was to determine the preferable angle of sloped walls for the proposed type $\mathrm{C}$ canals, as well as water depths and flow velocities that enable frogs to easily escape. In this process, frog behavior and climbing ability were also investigated.

\section{Materials and methods}

\section{Sample}

Eighty-five frogs were captured by hand and with a net from paddy fields and agricultural canals in Tochigi Prefecture. Their body sizes (snout-vent length) ${ }^{11}$ ranged from 19 to $67 \mathrm{~mm}$. Body size was classified into three categories for convenience of description: small frogs ( $<30 \mathrm{~mm}, n=16)$, medium frogs (30-40 mm, $n=39$ ) and large frogs ( $>40 \mathrm{~mm}, n=30)$. The captured frogs were reared in three plastic boxes of 34-90 L during the experimental period. After all experiments, they were returned to their original habitat.

\section{Experimental device}

Figure 4 shows a diagram of the experimental device used to determine the most preferable slope and hydraulic conditions for frogs to escape from canals. For ease of setup and behavioral observations, the veneerboard experimental canal was $20 \mathrm{~cm}$ wide and $25 \mathrm{~cm}$ deep. The dimensions of the experimental canal were based on actual agricultural canals, which usually range from 20 to $50 \mathrm{~cm}$ in width and depth. The flow rate was set at $2 \mathrm{~L} / \mathrm{s}$ and was measured using a water gauge. Water circulated through the device with a water tank, a pump, a triangular weir, the experiment canal, a water-level control gate, and a feedback canal. Halfway through the canal, partially sloped walls that could be adjusted to tilt 30 , $45,60,75$, and 90 degrees were placed as an escape countermeasure. Preliminary experiments showed that the veneer-board sloped wall was not different from an equal- ly sloped concrete wall of a U-shaped flume, as in actual canals, with regard to the ease of climbing by frogs.

\section{Experimental conditions}

In this study, (1) the preferable angle of slope for walls that frogs can easily climb (experiment 1) and (2) the preferable water depth and average flow velocity that urge the frogs to reach the sloped walls (experiment 2) were investigated. The preferable water depth and flow velocity of the canal are the conditions where frogs are more highly urged to go toward and reach the sloped walls. Table 2 shows the conditions of experiment 1 (cases $1-1$ to $1-15$ ) and experiment 2 (cases $2-1$ to $2-6$ ).

For cases 1-1 to 1-5, the canals were set up under dry condition. For cases $1-6$ to $1-15$, the flow rate was set at $2 \mathrm{~L} / \mathrm{s}$ based on field data collected where the frogs were captured. Water depth for cases 1-6 to 1-10 was set at $2 \mathrm{~cm}$ in which all frog could stand on the bottom of the canal and for cases $1-11$ to $1-15$ it was set at $5 \mathrm{~cm}$ in which no frogs could stand on the bottom of the canal. The water-level was controlled by a gate placed downstream (Fig. 4) to keep $2 \mathrm{~cm}$ and $5 \mathrm{~cm}$ at the point of the rectangle section. Angles of the sloped walls were set at 30, 45, 60, 75, and 90 degrees. Average flow velocities for water depths of 2 and $5 \mathrm{~cm}$ (Table 2) were 50 and 20 $\mathrm{cm} / \mathrm{s}$, respectively, calculated from the flow rate $(2 \mathrm{~L} / \mathrm{s})$, the water depth and the width of the canal. Preliminary tests of the actual distribution of surface flow velocity was measured by trajectory video records of 100 plastic float balls with a diameter of $5 \mathrm{~mm}$ released in the device, and the distribution was monitored using Flow-PTV software (Library Ltd., Tokyo, Japan). Figure 5 shows the flow velocity distribution at the water surface of the canal in case 1-6 (flow rate $2 \mathrm{~L} / \mathrm{s}$, water depth $2 \mathrm{~cm}$, wall angle 30 degrees) and case 1-11 (2 L/s, 5 cm, 30 degrees) as ex-

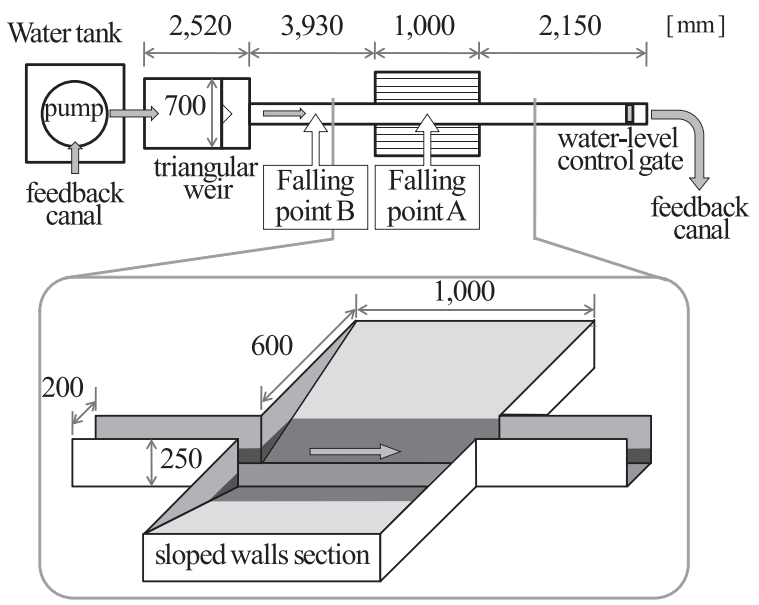

Fig. 4. Diagram of experimental device 
Table 2. Experimental conditions

\begin{tabular}{|c|c|c|c|c|c|}
\hline Case & $\begin{array}{l}\text { Flow } \\
\text { rate } \\
\mathrm{L} / \mathrm{s}\end{array}$ & $\begin{array}{l}\text { Depth } \\
\mathrm{cm}\end{array}$ & $\begin{array}{l}\text { Average flow } \\
\text { velocity }{ }^{1)} \\
\mathrm{cm} / \mathrm{s}\end{array}$ & $\begin{array}{l}\text { Degree of } \\
\text { sloped } \\
\text { walls }\end{array}$ & $\begin{array}{l}\text { Falling } \\
\text { point }^{2)}\end{array}$ \\
\hline $1-1$ & & & & 30 & \\
\hline $1-2$ & & & & 45 & \\
\hline $1-3$ & 0 & 0 & 0 & 60 & A \\
\hline $1-4$ & & & & 75 & \\
\hline $1-5$ & & & & 90 & \\
\hline $1-6$ & & & & 30 & \\
\hline $1-7$ & & & & 45 & \\
\hline $1-8$ & 2 & 2 & 50 & 60 & B \\
\hline $1-9$ & & & & 75 & \\
\hline $1-10$ & & & & 90 & \\
\hline $1-11$ & & & & 30 & \\
\hline $1-12$ & & & & 45 & \\
\hline $1-13$ & 2 & 5 & 20 & 60 & B \\
\hline $1-14$ & & & & 75 & \\
\hline $1-15$ & & & & 90 & \\
\hline $2-1^{3)}$ & 0 & 0 & 0 & 30 & A \\
\hline $2-2$ & 0.2 & 2 & 5 & & \\
\hline $2-3$ & 0.8 & 2 & 20 & & \\
\hline $2-4^{4)}$ & 2 & 2 & 50 & 30 & B \\
\hline $2-5$ & 0.5 & 5 & 5 & & \\
\hline $2-6^{5)}$ & 2 & 5 & 20 & & \\
\hline
\end{tabular}

1): Average flow velocity of rectangle section was calculated from the flow rate, the water depth and the breadth of the canal.

2):A, the center of the sloped wall section; B, $1 \mathrm{~m}$ above the sloped wall section (refer to Fig. 1).

3):Data of case 1-1 was used.

4):Data of case 1-6 was used.

5):Data of case 1-11 was used. amples of the measurement results. Average surface flow velocity in case 1-6 was calculated as $25 \mathrm{~cm} / \mathrm{s}$ with a range of $10-55 \mathrm{~cm} / \mathrm{s}$, and that in case 1-11 was $11 \mathrm{~cm} / \mathrm{s}$ with a range of $10-29 \mathrm{~cm} / \mathrm{s}$. Zones of parallel flow vectors with $40-60 \mathrm{~cm} / \mathrm{s}$ for case $1-6$ and $20-40 \mathrm{~cm} / \mathrm{s}$ for case 1-11 were distributed in the central part of the canal, and zones of $20 \mathrm{~cm} / \mathrm{s}$ or less were distributed outside of the zones in both cases. A reverse slow-flow vector and cross-flow vector toward the shore were observed near the foot of the sloped walls. Expansion of the canal width forms an artificial inlet with shallow water depth and slow water flows around the partially broadened spaces beside the sloped walls.

The conditions of experiment 2 (cases $2-1$ to $2-6$ ) were set up to investigate the preferable water depth and flow velocity of the canal in which frogs more easily go toward and reach the foot of sloped walls (Table 2). Angle of the sloped walls was kept at 30 degrees based on the results of experiment 1 , which showed the walls with slopes of 30 degrees allowed the frogs to escape most easily. Water depth was set at 2 or $5 \mathrm{~cm}$ and flow velocity was set at 5,20 or $50 \mathrm{~cm} / \mathrm{s}$ by combinations of flow rates $0.2,0.5,0.8$, and $2 \mathrm{~L} / \mathrm{s}$ and water depth. Moreover, the dry condition was tested. However, the water depth of 5 $\mathrm{cm}$ and flow velocity of $50 \mathrm{~cm} / \mathrm{s}$ was not tested due to over-capacity of the pump. The data of cases 1-1, 1-6 and 1-11 were respectively assigned to cases 2-1, 2-4 and 2-6 because they were exactly the same conditions.

\section{Methods}

After setting each condition (Table 2), 45 frogs randomly chosen from 85 sample frogs were put into a plastic carrying case and then concurrently allowed to fall

b) Case 1-11: flow rate of $2 \mathrm{~L} / \mathrm{s}$, water depth of $5 \mathrm{~cm}$ and sloped walls of 30 degrees

a) Case 1-6: flow rate of $2 \mathrm{~L} / \mathrm{s}$, water depth of $2 \mathrm{~cm}$ and sloped walls of 30 degrees



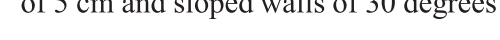

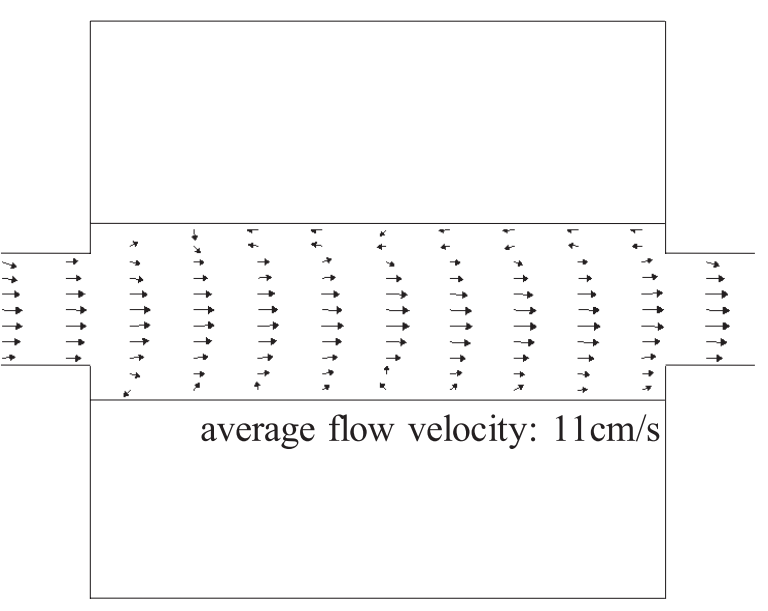

Fig. 5. Examples of the flow velocity at the water surface 
from the case into the experimental canal at point A or B of the canal (Fig. 4). For the dry conditions (cases 1-1 to 1-5 and case 2-1), the individuals fell into the center of the sloped wall section (Point A). When water was running (cases $1-6$ to $1-15$ and cases $2-2$ to $2-6$ ), the individuals were allowed to fall $1 \mathrm{~m}$ above the sloped wall section (Point B) because individuals tended to move downstream. The experimental duration was one hour and behavior of each individual during the experiment was filmed with a video camera located immediately above the canal. The body lengths of the individuals that escaped from the canal during each test were measured. After the experiments, all individuals were removed from the canal and returned to the rearing boxes. The experiment period was between August 22 and October 30, 2007. The room temperature was an average of 24.6 degrees $\mathrm{C} \pm 3.2 \mathrm{SD}$, the water temperature was 23.1 degrees $\mathrm{C} \pm 1.7 \mathrm{SD}$.

\section{Analysis}

The video records during all tests were carefully analyzed to observe the behavioral characteristics of individuals after falling. The proportion of frogs that reached the sloped walls and the proportion of frogs that escaped from the canal via the sloped walls were counted as indicators of the efficiency of the escape countermeasure. Henceforth, the indicators were described as 'proportion reaching' and 'proportion escaping' respectively.

\section{Results}

\section{Behavior of individuals after falling into the canal}

Behaviors of all individuals (45 frogs by 18 cases) were observed in the experiments. After falling into the

a) Case 1-6: flow rate of $2 \mathrm{~L} / \mathrm{s}$, water depth of $2 \mathrm{~cm}$ and sloped walls of 30 degrees

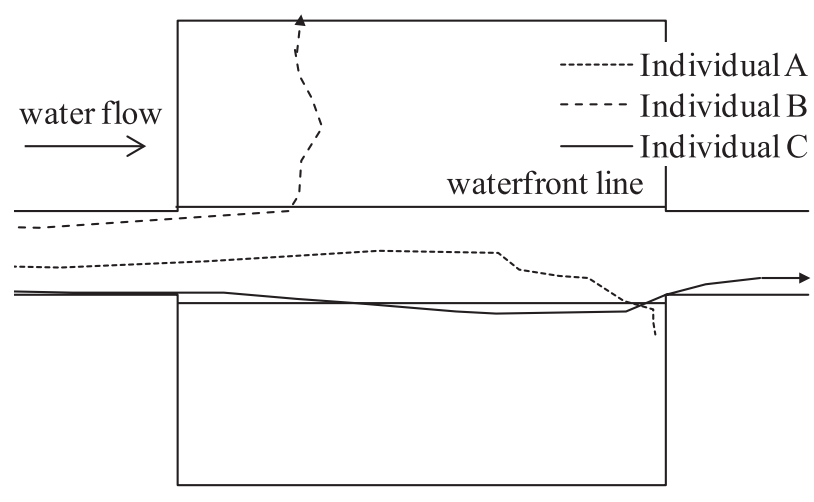

experimental canal, all individuals gradually scattered from the falling point. When the flow rate was $2 \mathrm{~L} / \mathrm{s}$ and the water depth was $2 \mathrm{~cm}$ (cases $1-6$ to $1-10$ ) or $5 \mathrm{~cm}$ (cases 1-11 to 1-15), most individuals immediately moved downstream. During movement downstream, the individuals often attempted to swim against the stream. The swimming behavior was composed of three processes: 1) individuals kicked with both legs together, 2) timing for kicking of each leg gradually became unsynchronized until they alternated between legs and 3) kicking stopped. Individuals kicking with both legs together was thought to be the most basic swim behavior and continued for only up to 11 seconds. Sometimes individuals temporarily stopped moving to rest by standing on the bottom if they could reach the bottom or holding on to the wall of the canal if they could not reach the bottom. However, they did not remain in one position for a long time.

The individuals showed various behaviors after reaching the sloped walls (Fig. 6). Some individuals stopped climbing halfway and settled until the end of the experiment (individual A of case 1-6, Fig. 6a) or swam again (individual $\mathrm{C}$ of case 1-6, Fig. 6a) after reaching the sloped walls. The individuals seldom swam upstream once they had moved downstream beyond the sloped walls. Consequently, of all tested individuals, 241 (30\%) escaped from the canal by using the sloped walls. Only 13 individuals (2\%) were observed leaping out of the canal, indicating the difficulty for $R$. $p$. porosa to escape from even small canals without an escape countermeasure.

\section{Preferred angle of the sloped walls}

Figure 7 shows the proportions of frogs reaching the slopes and the proportion of size-dependent individuals

b) Case 1-11: flow rate of $2 \mathrm{~L} / \mathrm{s}$, water depth of $5 \mathrm{~cm}$ and sloped walls of 30 degrees

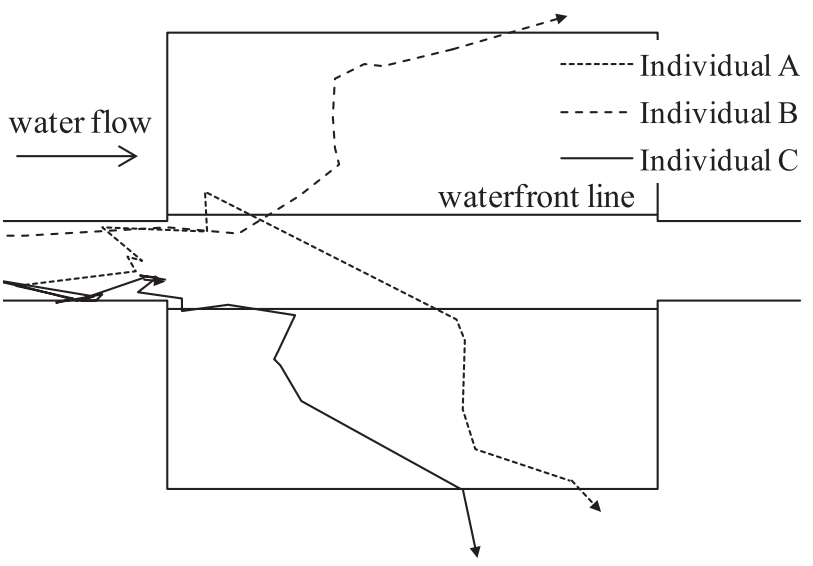

Fig. 6. Examples of the trajectories of individuals after falling into the canal 
a) Flow rate of $0 \mathrm{~L} / \mathrm{s}$ and depth of $0 \mathrm{~cm}$ (dry conditions)

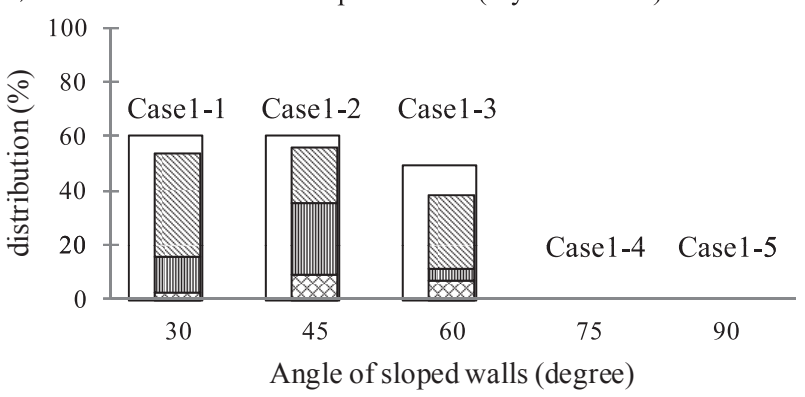

b) Flow rate of $2 \mathrm{~L} / \mathrm{s}$ and depth of $2 \mathrm{~cm}$

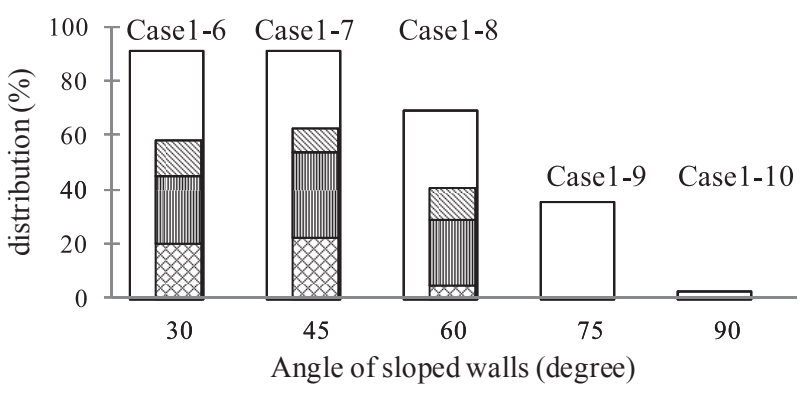

c) Flow rate of $2 \mathrm{~L} / \mathrm{s}$ and depth of $5 \mathrm{~cm}$



Fig. 7. Proportion of individuals reaching the sloped walls and proportion of size-dependent individuals escaping from the canal after falling into the canal a) flow rate of $0 \mathrm{~L} / \mathrm{s}$ and depth of $0 \mathrm{~cm}$, b) flow rate of $2 \mathrm{~L} / \mathrm{s}$ and depth of $2 \mathrm{~cm}$ and c) flow rate of $2 \mathrm{~L} / \mathrm{s}$ and depth of $5 \mathrm{~cm}$

$\square$ : Individuals that reached the sloped walls.

$\mathbb{N}$ : Individuals with snout-vent length of less than $30 \mathrm{~mm}$ that escaped through the sloped walls.

Ind : Individuals with snout-vent length of 30 to 40 mm that escaped through the sloped walls.

: Individuals with snout-vent length of over 40 $\mathrm{mm}$ that escaped through the sloped walls.

escaping from the canal after falling into the canal on the conditions of a) flow rate of $0 \mathrm{~L} / \mathrm{s}$ and depth of $0 \mathrm{~cm}, \mathrm{~b}$ ) flow rate of $2 \mathrm{~L} / \mathrm{s}$ and depth of $2 \mathrm{~cm}$, and c) flow rate of 2 $\mathrm{L} / \mathrm{s}$ and depth of $5 \mathrm{~cm}$. For the dry conditions (cases 1-1 to $1-5$ ), $38 \%$ (case $1-3$ ) to $56 \%$ (case $1-2$ ) escaped when the slope angle was 30-60 degrees (Fig. 7a). However, both proportions reaching and proportions escaping were $0 \%$ when the slope angle was 75-90 degrees (cases 1-4 to 1-5). Nine large individuals leaped out of the canal when the slope angle was 45-90 degrees.

With running water (cases 1-6 to 1-15), all individuals that escaped from the canal climbed the sloped walls, namely no individuals leaped out of the canal. The proportion escaping from the canal did not differ among the three size classes, and there was little difference between the canals with a depth of $2 \mathrm{~cm}$ (cases 1-6 to 1-10, Fig. 7b) and $5 \mathrm{~cm}$ (cases 1-11 to 1-15, Fig. 7c) in the proportion reaching and the proportion escaping from the sloped walls at each angle. Proportions reaching were 69\% (case 1-8) to 91\% (cases 1-6 and 1-7) and proportions escaping were $20 \%$ (case $1-13$ ) to $62 \%$ (case $1-7$ ) when the slope angle was 30-60 degrees. On the other hand, no individuals climbed to the top of the walls with slopes of 75-90 degrees even though some individuals reached the sloped walls (cases $1-9$ to $1-10$ and $1-14$ to $1-15$ ). The differences between proportions reaching with 30 degree sloped walls and those of 45 degree sloped walls were small, and the proportion escaping showed a similar tendency. However, eight large individuals finally were able to climb to the top of the 45 degree wall after repeatedly slipping down and climbing up several times.

\section{Preferred water depth and flow velocity}

Figure 8 shows the proportion reaching and proportion escaping after falling into the canal of cases $2-1$ to 2-6: a dry condition and combinations of depths of 2 and $5 \mathrm{~cm}$, and average flow velocities of 5,20 and $50 \mathrm{~cm} / \mathrm{s}$. For the dry condition (case 2-1), the proportion reaching and the proportion escaping were $60 \%$ and 53\%, respectively. With running water (cases $2-2$ to $2-6$ ), the proportion reaching and the proportion escaping from the canal with a water depth of $2 \mathrm{~cm}$ and flow velocity of 5 $\mathrm{cm} / \mathrm{s}$ (case 2-2) were much lower than those of the canal with the other combinations of water depth and flow velocity (cases 2-3 to 2-6). The proportion reaching the sloped walls with a water depth of $2 \mathrm{~cm}$ and flow velocity of $5 \mathrm{~cm} / \mathrm{s}$ was $4 \%$ (case 2-2), whereas those under the other combinations were $78 \%$ (case 2-5) to $91 \%$ (case $2-4)$. The proportion escaping of the former was $2 \%$ (case 2-2), whereas those of the latter were 33\% (case $2-3)$ to $58 \%$ (case $2-4$ ). There were 4 large individuals that leaped out of the canal (cases 2-2 and 2-5).

\section{Discussion}

\section{Preferred angle of sloped walls}

Most individuals were equally able to reach and escape from canals with sloped walls of 30 degrees and 45 degrees (Fig. 7). However the large individuals often slipped off when they were climbing the walls with slopes of 45 degrees, indicating this slope is probably not good 
for large frogs. If they can leap out of the canal, concern for large frogs may be less important. Actually, the individuals with snout-vent length of 38-62 mm leaped out of the canal in this study. In this regard, the vertical jumping ability of $R$. p. porosa or other related frogs has not been studied. However, Doi ${ }^{2}$ reported that the horizontal jumping ability of $R$. nigromaculata and $R$. p. brevipoda, which are extremely closely related to $R$. p. porosa, were positively correlated with their body length. The vertical jumping ability of $R$. p. porosa may also increase for larger frogs. Even so, canals with deeper walls or deep-water canals for which $R$. p. porosa cannot stand on the bottom probably prevent them from leaping out. In our experiments, 241 individuals (30\%) escaped from the canal by using the sloped walls and only 13 individuals (2\%) escaped by leaping out of the canal, indicating the difficulty for $R$. $p$. porosa to escape from even the small canals without an escape countermeasure. Therefore, the more efficient countermeasure to enable frogs to climb regardless of body size should design the angle of sloped walls to be lower than 30 degrees. In addition, frogs might be able to climb more steeply sloped walls if the wall surface is rough enough to prevent slipping, but further experiments using another material for the sloped walls will be necessary to test this.

Okochi et al. ${ }^{16}$ reported that newly-metamorphosed Bufo japonicus formosus, which also have no adhesive discs, can climb sloped walls of less than 60 degrees, and escape more easily from a canal with a gentler slope. The climbing ability of $R$. p. porosa in this study appears similar to that of $B$. $j$. formosus so frogs that lack adhesive discs may have a similar climbing ability. The climbing ability of other species should be investigated in order to determine the angle of a sloped wall that enables various frogs to easily escape.

\section{Preferred water depth and flow velocity}

In the canal for cases 2-3 to 2-6, most individuals moved downstream, reached and climbed the sloped walls (Fig. 8). However, few individuals reached the sloped walls when the water depth was $2 \mathrm{~cm}$ and flow velocity was $5 \mathrm{~cm} / \mathrm{s}$ (case 2-2). In this case, the water was shallow and the flow rate was slow so that all individuals freely moved or remained in the canal and did not go downstream to reach the sloped walls. Similarly, few individuals reached the sloped walls in the dry canal (case 2-1) even when they fell into the center of the canal near the sloped walls. These findings may indicate that frogs do not attempt to go toward the sloped walls to escape from the canal when they can move freely. Observations of the individuals and the results of supplementary experiments ${ }^{18}$ showed that $R$. p. porosa swim shoreward when their toes do not reach the bottom of the canal. Moreover, a reverse slow-flow vector and cross-flow vector toward the shore were observed near the foot of the sloped walls (Fig. 5), which probably increased the chances of individuals reaching the sloped walls. Consequently, these hydraulic conditions may encourage individuals falling into a deep-water canal or a fast flow canal to move downstream and reach the sloped walls, whereas the hydraulic conditions of shallow and slow water give individuals falling into canals a free hand so that they did not reach the escape countermeasure slope. Therefore, adjusting the hydraulic conditions to deeper-water ( $\geqq 5 \mathrm{~cm}$ ) or faster flow ( $\geqq 20 \mathrm{~cm} / \mathrm{s}$ ) will help frogs reach the escape countermeasures. In these experiments, very few individuals swam upstream after they had gone downstream beyond the sloped walls. We observed in several field surveys that $R$. $p$. porosa was often swept downstream in agricultural canals. These observations indicate that $R$. $p$. porosa is not good at swimming in running water. Therefore, the water depth rather than the flow velocity should be controlled. Especially near the escape countermeasures, the flow velocity should be as low as possible to prevent frogs from being washed away.

\section{Conclusion}

We propose a partial concrete canal with gently sloped walls as countermeasures for frogs to escape the canal. The preferred angle of sloped walls, water depth and flow velocity that enable frogs to escape from agri-

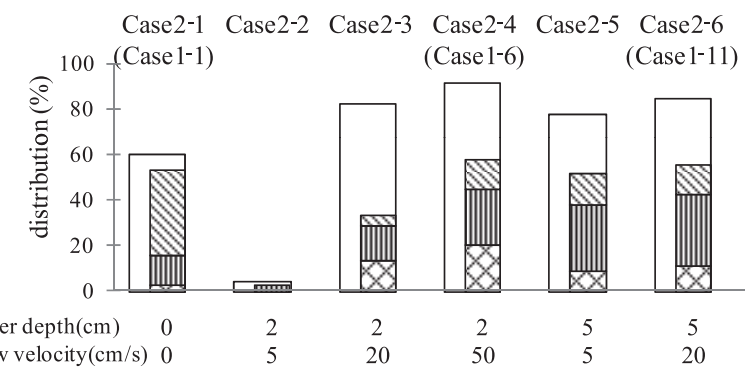

Fig. 8. Proportion of individuals reaching the sloped walls and proportion of individuals escaping from the canal after falling into the canal with combinations of depths of 0,2 and $5 \mathrm{~cm}$ and average flow velocities of $0,5,20$ and $50 \mathrm{~cm} / \mathrm{s}$

$\square$ : Individuals that reached the sloped walls.

$\mathbb{N}$ : Individuals with snout-vent length of less than $30 \mathrm{~mm}$ that escaped through the sloped walls.

$\mathbb{\| l}$ : Individuals with snout-vent length of 30 to 40 $\mathrm{mm}$ that escaped through the sloped walls.

: Individuals with snout-vent length of over 40 $\mathrm{mm}$ that escaped through the sloped walls. 
cultural canals with deep concrete walls were investigated for designing the countermeasures. We conclude the following: (1) the angle of the sloped walls should be less than 30 degrees for frogs to easily climb without slipping; (2) adjusting water depth to $5 \mathrm{~cm}$ or more, a depth at which most frogs cannot stand on the bottom, will assist escape because frogs are not able to move freely and are encouraged to swim toward the downstream escape countermeasures; and (3) flow velocity should be lower around the countermeasures because the frogs are not good at long-distance swimming and cannot remain under running water a long-time. The partial concrete canal with gently sloped walls is a practical method to be used with low flow velocity near the shore of the sloped wall.

To develop more sufficient countermeasures, information on the behavior and physical characteristics of not only $R$. p . porosa but also other species should be determined and applied. Moreover, on-site adjustments and monitoring can enhance the effectiveness of the countermeasures.

\section{Acknowledgments}

We thank Messrs. J. Terakawa, J. Shinozaki and M. Ishijima for developing the experimental device and Drs. M. Mori and T. Nakaya for helping with the experiments.

\section{References}

1. Doi, T. (2001) Climbing ability of Daruma Pond Frog, Rana porosa brevipoda. Ryoseiruishi (Amphibian History), 6, 25-27 [In Japanese].

2. Doi, T. (2002) How much is the difference in jumping ability between Black-spotted Pond Frog, Rana nigromaculata and Daruma Pond Frog, Rana porosa brevipoda? Ryoseiruishi (Amphibian History), 8, 12-16 [In Japanese].

3. Fujioka, M. \& Lane, S. J. (1997) The impact of changing irrigation practices in rice fields on frog populations of the Kanto Plain, central Japan. Ecol. Res., 12, 101-108.

4. Hasegawa, M. (1995) Rana japonica in valley-bottom paddy fields in the Kanto Plain, Japan. In Minami kanto no shizenshi (Natural History of the Southern Kanto Plain), eds. Osawa M. \& Ohara T., Asakura-shoten, Tokyo Japan, 105-112 [In Japanese].

5. Hasegawa, M. (2003) Techniques in surveying organisms for agricultural engineers (8): Field study of amphibians. Nogyo doboku gakkai shi (J. Jpn. Soc. Irrig. Drain. Reclam. Eng.), 71(5), 57-62 [In Japanese].

6. Hirai, T. \& Matsui, M. (2001) Food habits of an endangered Japanese frog, Rana porosa brevipoda. Ecol. Res., 16(4), 737-743.
7. Ishizuka, S. \& Suzuki, Y. (1998) Modification of side drainage ditch block for amphibian escape. Kochi daigaku nogakubu enshurin hokoku (Bull. Kochi Univ. For.), 25, 199-208 [In Japanese with English summary].

8. Kawanishi, E. \& Nishihara, M. (1995) Escape test of small animals upon countermeasure of U-shaped side ditch with consideration to small animals. Kankyou joho kagaku ronbunshu (Pap. Environ. Inf. Sci.), 8, 57-62.

9. Kosugi, A. (1960) On the food habits of some herons. Yamashina chorui kenkyujo kenkyu hokoku (J. Yamashina Inst. Ornithol.), 2(2), 23-32 [In Japanese with English summary].

10. Lane, S. J. \& Fujioka, M. (1998) The impact of changing irrigation practices on the distribution of foraging egrets and herons (ARDEIDAE) in the rice fields of central Japan, Biol. Conserv., 83(2), 221-230.

11. Maeda, N. \& Matsui, M. (1989) Nihon kaeru zukan (Frogs and toads of Japan). Bun-Ichi Sogo Shuppan, Tokyo [In Japanese].

12. Matsuzawa, S. et al. (2008) Verification of population density and distribution of frogs inhabiting ecologically sound land consolidated field. Nogyo noson ko gakkai taikai koenkai koen yoshishu (Translation), 704-705 [In Japanese].

13. Mattoon, A. (2002) Deciphering amphibian declines. In State of the World 2001: A Worldwatch Institute Report on Progress Toward a Sustainable Society (State of the World), eds. Brown, L. R. et al., W. W. Norton \& Co. Inc., USA, 63-82.

14. Mizutani, M., Takahashi, N. \& Hayashi, M. (2005) Effects of covering the small-scale concrete ditch with a wooden lid on inhabitation of Rana japonica. Nogyo doboku gakkai ronbunshu (Trans. Jpn. Soc. Irrig., Drain. Rural Eng.), 73(1), 77-78 [In Japanese].

15. Nogami, H. \& Suzuki, Y. (1999) The actual condition that amphibians drop into ditch block and test of slope ditch block for amphibian escape. Kochi daigaku nogakubu enshurin hokoku (Bull. Kochi Univ. For.), 26, 13-23 [In Japanese with English summary].

16. Okochi, I., Okawabata, O. \& Kurashina, N. (2001) Prevention of amphibian mortality in roadside ditch. Nihon ringakkaishi (J. Jpn. For. Soc.), 83(2), 125-129 [In Japanese with English summary].

17. Sato, T. \& Azuma, A. (2004) Frogs abundance and environments of levees relationships around paddy fields in alluvial fan. Randosukepu kenkyu (J. Jpn. Inst. Landsc. Archit.), 67(5), 519-522 [In Japanese with English summary].

18. Watabe, K. et al. (2009) Fundamental experiment to determine escape countermeasures for frogs falling into agricultural canals. Nogyo noson ko gakkai ronbunshu (Trans. Jpn. Soc. Irrig., Drain. Rural Eng.), 77(5), 15-21 [In Japanese].

19. World Conservation Union Species Survival Commission (2005) Amphibian conservation action plan, Proceedings IUCN/SSC amphibian conservation summit 2005. http:// amphibiaweb.org/declines/acap.pdf. 